\title{
A new tool for experimental tumour research
}

\author{
CHENGWEN ZHANG ${ }^{1}$, NIKOLAUS GASSLER ${ }^{2}$, PHILIPP A. SCHNABEL ${ }^{2,3}$, HENDRIK DIENEMANN ${ }^{3}$, \\ ARMIN KOLB $^{4}$, PETER BUCHLER ${ }^{4}$, MARKUS BUCHLER $^{4}$, HELMUT FRIESS $^{4}$ and INGRID HERR ${ }^{1,5}$ \\ ${ }^{1}$ Molecular Urooncology, German Cancer Research Center; ${ }^{2}$ Department of Pathology, ${ }^{3}$ Thoraxklinik-Heidelberg, \\ Departments of ${ }^{4}$ General Surgery, ${ }^{5}$ Urology, University of Heidelberg, Heidelberg, Germany
}

Received November 2, 2005; Accepted February 1, 2006

\begin{abstract}
Surgical resected tumours are often stored for hours in the clinic upon transfer to the bench leading to apoptosis of tumour cells making them no longer suitable for molecular analysis and diagnostic procedures. The way out of this problem may be a new oxygen-enriched solution (OES). We tested this agent using surgical resections of carcinomas of lung, rectum and pancreas. Immediately after resection, one part of each individual tumour was stored in PBS and the other part in OES, and the content of viable or dead cells was determined by trypan blue exclusion and MTT-assay. We found that OES keeps tumour cells up to 3 days and longer more viable than PBS and reduces the percentage of dead cells without inducing therapy resistance and affecting the outcome of experimental procedures. Thus, storing freshly resected tumours in OES may save time for tumour transfer and initiation of experiments.
\end{abstract}

\section{Introduction}

Thousands of patients with solid tumours undergo surgery each year. Depending on the tumour size, only parts of the surgical resections are needed for pathological examination. The remaining tumour pieces are discarded or are used for molecular diagnosis and basic research to define optimized treatment strategies in cancer. However, a main obstacle is the time needed for the transfer of the tumours from the clinic to the laboratory. As the patient care has priority, resected tumours are often stored for hours in the clinic before a clinician has time enough to initiate the transport to the basic researcher. During storing, many of the tumour cells undergo

Correspondence to: Professor Ingrid Herr, German Cancer Research Center, Molecular Urooncology, E095, Im Neuenheimer Feld 280, 69120 Heidelberg, Germany

E-mail: i.herr@dkfz.de

\section{Abbreviations: OES, oxygen-enriched solution}

Key words: surgical resection, tumour specimen, lung, pancreatic, rectum carcinoma, apoptosis, viability apoptosis and thus are no longer suitable for molecular analysis. The way out of this problem may be a new oxygenenriched and serum-free solution (OES) containing inorganic salts, amino acids, vitamins, cholesterol, adenosine and low protein. The speciality of OES is proposed to be the transport of oxygen which is available on time when needed.

Prior to performing a huge prospective study using freshly resected tumours from patients, we tested the usefulness of OES for transport and preservation of specimens. The promising results that we obtained with OES are presented in the present study. Due to the good results, OES was in turn used for transport and conservation of tumours throughout the prospective study from which parts are already published (1-4).

\section{Materials and methods}

Oxygen-enriched solution (OES). OES is a new product kindly provided by F. Bach (Onco Science, Wedel, Germany) for investigation. This solution contains inorganic salts, aminoacids, vitamins, cholesterol and adenosine, low protein and is serum-free. The speciality is to transport oxygen which is available on time when needed. In case of cryoconservation, there are no further ingredients needed like DMSO or HES because the solution does not build crystals when the freezing protoid developed by Onco Science is being used. Since the detailed composition of OES is patented, further informations may be obtained by F. Bach.

Surgical resected tumours. Solid tumours were resected, transported in PBS or OES to the laboratory where the fresh tissues were minced in RPMI medium supplemented with $20 \%$ heatinactivated fetal bovine serum (Sigma, Deisenhofen, Germany), $25 \mathrm{mM}$ HEPES, $2 \mathrm{mM}$ L-glutamine and Pen/Strep (all from Gibco/Life Technologies, Paisley, Scotland) under sterile conditions. Patient material was obtained under the approval of the ethics committee of the University of Heidelberg. Diagnoses were established by conventional, clinical and histological criteria according to the World Health Organization (WHO). All surgical resections were indicated by principles and practice of oncological therapy.

Cisplatin. A stock solution of cytotoxic cisplatin (Sigma) was prepared in DMSO and final concentrations of the solvent in medium were $\leq 0.01 \%$. 
A

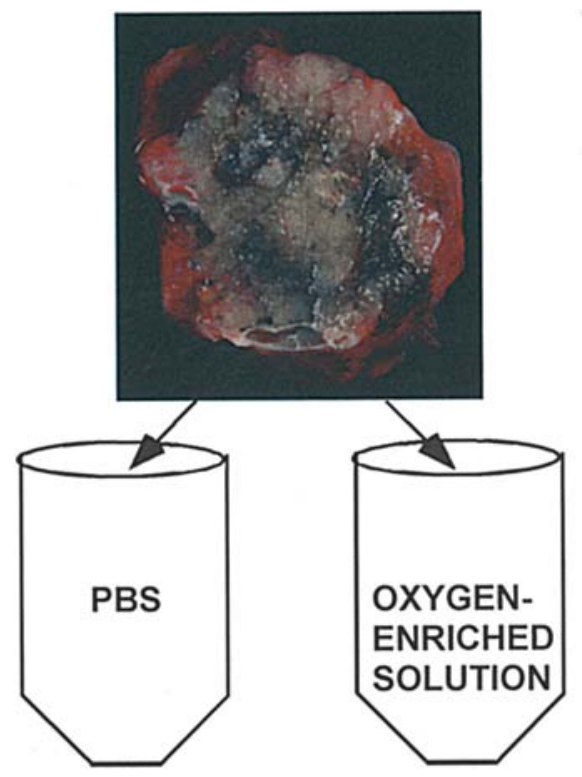

B

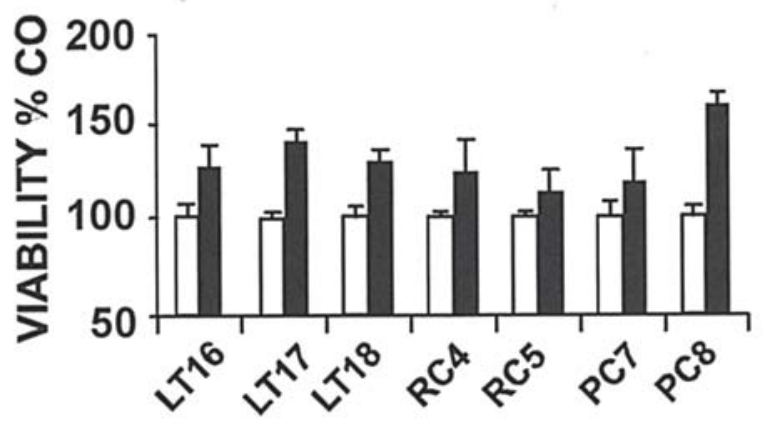

Figure 1. OES enhances viability of lung, rectal and pancreatic tumours after storage for $4 \mathrm{~h}$ and compared to PBS (A). Two pieces of each freshly resected tumour derived from three lung (LT16, LT17, LT18), two rectal (RC4, RC5) and two pancreatic carcinomas (PC7, PC8) were stored in $10 \mathrm{ml}$ PBS (white bars) or $10 \mathrm{ml}$ OES (black bars) at $4^{\circ} \mathrm{C}$ for $4 \mathrm{~h}(\mathrm{~B})$. Thereafter, tumour cells were isolated and viability was measured by the MTT-assay. Mean values \pm SD are shown.

MTT-assay. Primary tumour cells were resuspended at $5 \times 10^{5} / \mathrm{ml}$ in 96 -well microplates, $100 \mu 1$ per well. After treatment the MTT-assay was performed as described (1-4).

\section{Results}

To examine the usefulness of OES for keeping freshly resected tumours viable longer, we analyzed three lung tumours, two rectal tumours and two pancreatic tumours of patients. Immediately after resection, parts of the individual tumours, which were not needed for pathological analysis, were stored in tubes containing either $10 \mathrm{ml}$ PBS or $10 \mathrm{ml} \mathrm{OES} \mathrm{at} 4^{\circ} \mathrm{C}$ (Fig. 1A). After $4 \mathrm{~h}$, tumour cells were isolated and viability was examined by the MTT-assay. Strongly enhanced viability was found in all cells derived from OES compared to cells stored in PBS (Fig. 1B). To confirm this finding we counted viable and dead cells by trypan blue exclusion after storage in OES or PBS for 4 h, 3 and 7 days (Fig. 2). While no differences using this more insensitive method were found at $4 \mathrm{~h}$, more viable and less dead cells were clearly present in the OES-derived fractions at 3 and 7 days compared to cells

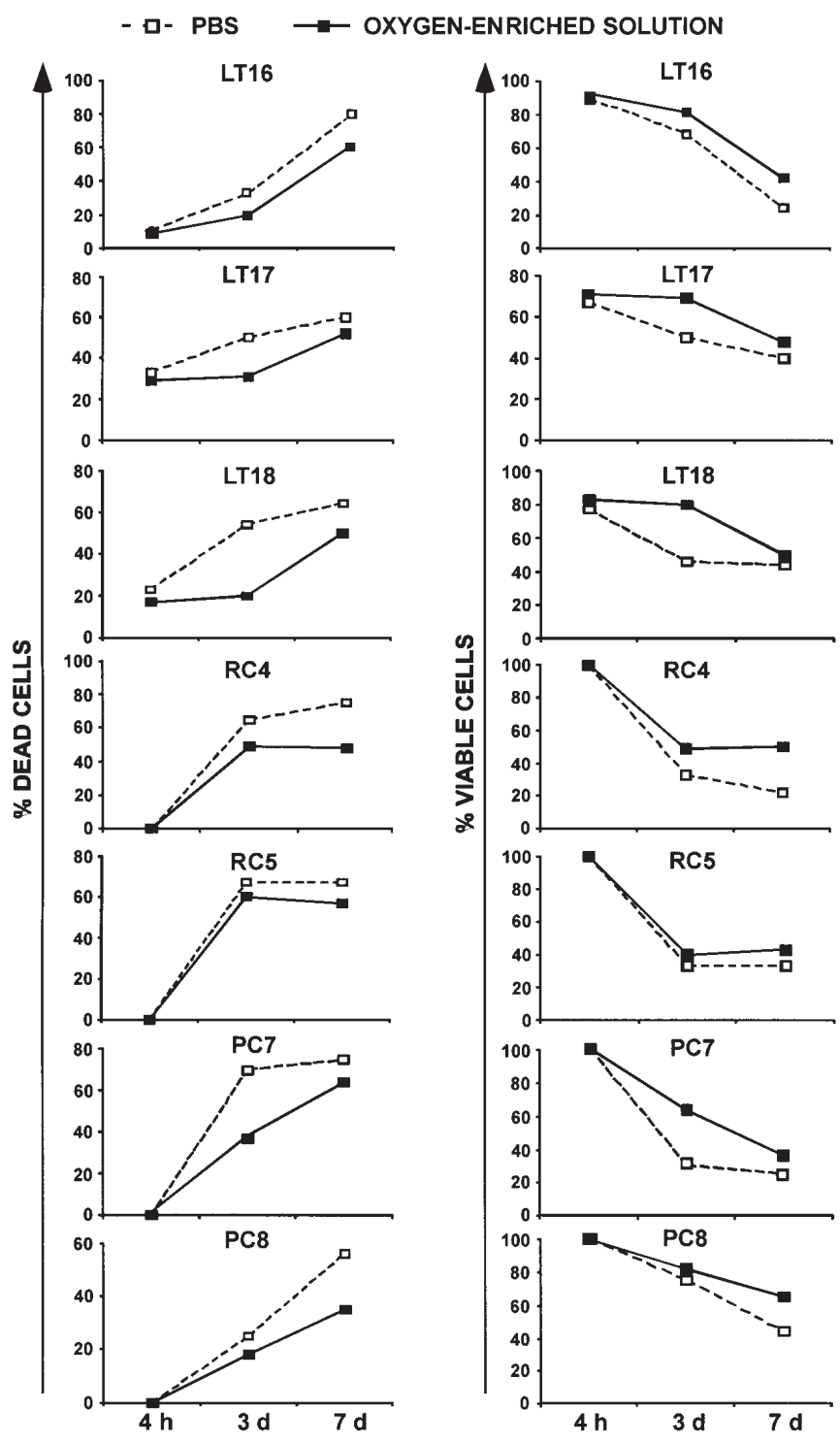

Figure 2. OES keeps tumour cells viable longer and reduces death up to seven days compared to PBS. Cells were isolated from the same carcinomas described above 4 h, 3 and 7 days after storage in OES or PBS and viable and dead cells were counted by trypan blue exclusion and microscopy.

derived from PBS. Thus, OES may be suited to keep tumours viable overnight or over the weekend at $4^{\circ} \mathrm{C}$, while it is less suited to store the cells longer than 3 days, since cells are dying in any case, although to a smaller extent than cells of tumours stored in PBS.

Another question is, whether OES might induce apoptosis resistance thereby keeping the cells viable longer. In case of inducing resistance, OES might interfere with the results of apoptosis assays and, therefore, may not be suited for preservation. We examined this point by treatment of cells, which have been stored in OES or PBS for $4 \mathrm{~h}$, with different concentrations of cisplatin. Viability was measured by the MTT-assay $24 \mathrm{~h}$ later (Fig. 3). While cells derived from OES exhibited an overall higher percentage of viability, the percentage of reduction in viability was comparable between cells stored in PBS or OES. Thus, induction of therapy resistance by OES may be excluded. 


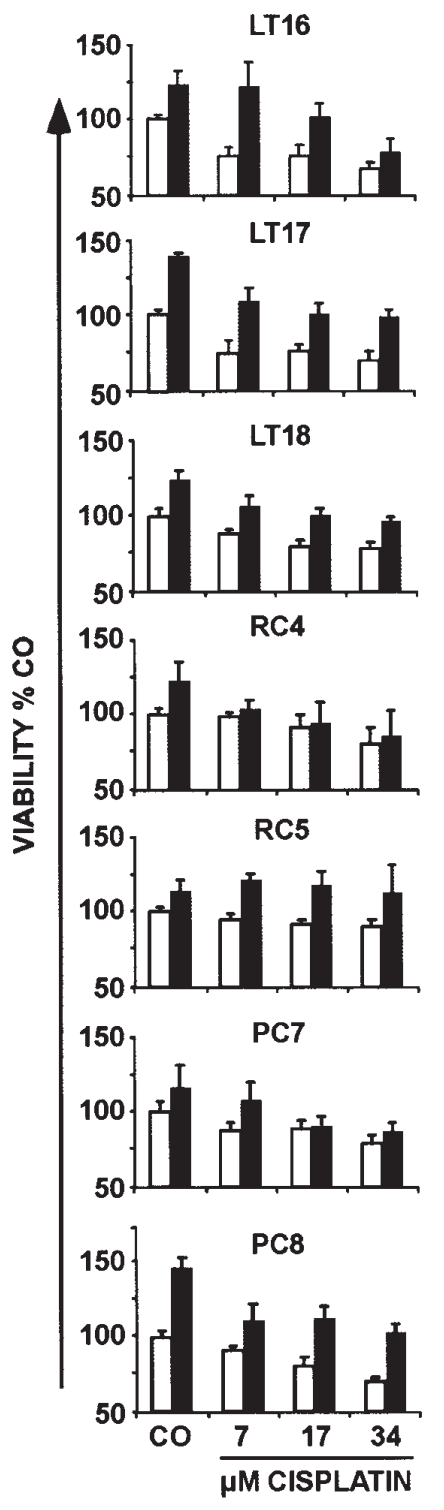

Figure 3. OES does not induce therapy resistance. Cells from tumours stored for $4 \mathrm{~h}$ in either OES or PBS were treated with cisplatin $(7,17,34 \mu \mathrm{M})$ or were left untreated (CO) and $24 \mathrm{~h}$ later viability was measured by the MTTassay. Mean values \pm SD are shown.

\section{Discussion}

Growing interest in proteomics and genomics of patient's tumours demand new strategies in preservation of the excised tumour-tissues, since the excision, transport and handling of the tissue biopsies are crucial. Therefore, we tested in the present study the suitability of a new oxygen-enriched solution (OES) proposed by the manufacturer to be useful in prolonging viability of freshly resected tumours. Immediately after surgical resection, tumour probes were divided in two pieces from which one was stored in OES and the other one in PBS, as usual. In this manner a higher percentage of viable cells was found in all examined biopsies derived from three lung, two rectum and two pancreatic carcinomas $4 \mathrm{~h}$ after storage in OES compared to PBS. Moreover, a clear difference was also seen 3 days after storage with the most pronounced protection in lung tumours. Even 7 days after storage, a difference in viability was seen in most of the examined tumours, although the percentage of dead cells increased and was high in both solutions.

The detailed mechanism by which OES preserves tumours much longer than PBS is not known, although the manufacturer suggests that this solution penetrates into the tissue and protects the RNA content of the whole sample for several days. Since this may be due to enhanced repair mechanisms, we examined induction of apoptosis resistance by OES. However, we found no hint of apoptosis resistance as tested by treating the isolated tumour cells of both fractions with cisplatin and measuring viability in control and drug-treated cells. The only visible difference was a higher basal viability of cells derived from OES, but the proportion of drug-reduced viability remained the same in OES- and PBS-conserved tumour cells.

One important characteristics of OES is its enrichment in inorganic salts, amino acids, vitamins, cholesterol and adenosine. Most of these substances function as biological redox systems and may thereby contribute to stabilization of enzymes, proteins, DNA and other cellular components. On the other hand, the above described ingredients of OES are also present in normal cell culture medium which do not act as conservative agents. Therefore, the most valuable attribute of OES may be its enrichment in oxygen bound to a carrier which sets it free when needed.

Together, and based on our presented results, OES may be suited for preserving freshly resected tumours viable longer than PBS and may save time for the initiation of experimental diagnostics and basic research.

\section{References}

1. Gassler N, Zhang C, Schnabel PA, Dienemann H, Debatin K-M, Mattern J and Herr I: Dexamethasone-induced cisplatin and gemcitabine resistance in lung carcinoma samples treated ex vivo. Br J Cancer 92: 1084-1088, 2005.

2. Zhang C, Kolb A, Gassler N, Wenger T, Herzer K, Debatin K-M, Buechler M, Friess H and Herr I: Dexamethasone desensitizes hepatocellular and colorectal tumours toward cytotoxic therapy. Cancer Lett (In press).

3. Zhang C, Marme A, Wenger T, Gutwein P, Edler L, Rittgen W, Debatin K-M, Altevogt P and Herr I: Glucocorticoid-mediated inhibition of chemotherapy in ovarian carcinomas. Int J Oncol 28: 551-558, 2006.

4. Zhang C, Mattern J, Haferkamp A, Pfitzenmaier J, Debatin K-M, Hohenfellner M, Rittgen W, Edler L, Groene E and Herr I: Corticosteroid-induced chemotherapy resistance in urological cancers. Cancer Biol Ther (In press). 